Holoprosencephaly is a congenital malformation dating to the first 4 weeks of embryogenesis and caused by a defect in patterning of the basal forebrain, with incomplete separation of the cerebral hemispheres and basal ganglia. The 3 classic subtypes of HPE, alobar, semilobar, and lobar, are based on the degree of hemispheric separation. A fourth subtype, $\mathrm{MIH}$, is characterized by nonseparation of the posterior frontal and parietal regions of the cerebral hemispheres, whereas more polar areas of the cerebrum, basal forebrain, anterior frontal lobes, and occipital regions, are fully cleaved. $\mathrm{MIH}$ cases also have normal separation of the lentiform nuclei and hypothalamus, correlating with the absence of choreoathetosis and normal endocrine function, including temperature regulation, in this subtype of HPE. The thalamus is the most commonly affected deep gray nucleus in $\mathrm{MIH}$, with nonseparation in $33 \%$ of cases. This mainly topographical classification of CNS malformations is being replaced by a new classification based on the integration of morphological and molecular genetic criteria, as proposed by Sarnat HB and Flores-Sarnat L, 2001 (see Ped Neur Briefs August 2001;15:57). MIH is thought to be caused by impaired induction or patterning of the embryonic roof plate, and is sometimes related to ZIC2 mutations.

In an editorial, Patterson MC of Columbia University, Neurological Institute, NY, emphasizes the associated facial dysmorphism as a reflection of the severity of the brain malformation in HPE (Holoprosencephaly, the face predicts the brain; the image predicts its function. Neurology Dec (2 of 2) 2002;59:1833-1834). Gross facial malformation such as a single eye (cyclopia) is predictive of the most severe alobar type of HPE, whereas mild to moderate facial dysmorphism (cleft lip or palate, hypertelorism) corresponds with the milder MIH subtype or an asymptomatic carrier state for HPE. A high incidence of delayed myelination has been found in children with classical HPE, whereas MIH cases have normal myelination, appropriate for age, as described in the following article (Barkovich AJ et al. 2002).

\title{
WHITE MATTER MATURATION IN HOLOPROSENCEPHALY
}

White matter maturation in holoprosencephaly (HPE) was assessed by MRI scans in 47 patients (age 1 day to 16 years, median age 7 months) evaluated at the University of California, San Francisco. White matter maturation was delayed in 25 of 47 patients, and was easier to detect in infants compared to older children (24/32 affected $<1$ year of age, $1 / 15>1$ year of age). The classic severe subtypes of HPE were especially involved ( $2 / 4$ lobar, 20/31 semilobar, 3/6 alobar), whereas 0/6 with MIH had myelin delay. The classic and $\mathrm{MIH}$ variants of HPE appear to have different patterns of myelination, reflecting different underlying causes. (Barkovich AJ, Simon EM, Glenn OA et al. MRI shows abnormal white matter maturation in classical holoprosencephaly. Neurology Dec (2 of 2) 2002;59:1968-1971). (Reprints: Dr AJ Barkovich, Division of Neuroradiology, L 371, University of California, 505 Parnassus Ave, Box 0628, San Francisco, CA 94143).

COMMENT. Infants with classic holoprosencephaly (HPE) have delayed white matter maturation. HPE results from abnormal development of the floor plate and ventral neural tube, at the level of the prosencephalon, and oligodendrocytes, involved in myelin production, originate in the ventral aspect of the neural tube close to the floor plate. These 
processes depend on induction by the protein sonic hedgehog $(\mathrm{SHH})$, which is mutated in some forms of familial HPE (Roessler E et al, 1996, cited by Barkovich, 2002). The finding of normal myelination in MIH supports the theory of a normal floor plate in this subtype of HPE, and separate genetic and environmental factors in etiology.

\section{TOXIC DISORDERS}

\section{NEUROLOGIC COMPLICATIONS OF STEM CELL TRANSPLANTS}

The incidence and risk factors of severe neurologic events (SNE) were evaluated in 272 consecutive children who received allogeneic or autologous hematopoietic stem cell transplantation (HSCT) for hematologic or nonhematologic diseases at the G Gaslini Children's Research Institute, Genova, Italy. Median age at transplant was 8.5 years (range, 2 months to 19.5 years); and median follow-up was 15 months (range, 2 days to 15.6 years). SNE developed after a median of 90 days (range, 5 days to 8.8 years) after HSCT in 37 children (13.6\%). Seizures occurred in $19(51 \%)$, impaired consciousness in $12(32 \%)$, involuntary movements in $3(8 \%)$, and miscellaneous SNE in 10. Symptoms were attributed to cyclosporine A (CSA) toxicity in 21 (54\% of all SNE), to irradiation or chemotherapy injury in $7(17 \%)$, CNS infection in $7(17 \%)$, CNS hemorrhages in $3(7 \%)$, and to immune-mediated complications in the remaining $2(5 \%)$. Four children had more than one SNE. Eleven (30\%) died because of neurologic complications. Risk factors for SNE included the type of HSCT (allogeneic vs autologous; $\mathrm{p}=0.002$ ); treatment with total body irradiation (TBI) $(\mathrm{p}=0.02)$; development of severe acute graft-vs-host disease (GvHD >grade 2); and treatment with CSA. (Faraci M, Lanino E, Dini G et al. Severe neurologic complications after hematopoietic stem cell transplantation in children. Neurology Dec (2 of 2) 2002;59:1895-1904). (Reprints: Dr Maura Faraci, Department of Hematology?Oncology, Bone Marrow Transplant Unit, G Gaslini Children's Research Institute, Largo G Gaslini, 5, 16147 Genova, Italy).

COMMENT. Severe neurologic complications (SNE), especially seizures and impairment of consciousness, may be expected in $14 \%$ of children receiving bone marrow transplants, and a mortality rate of $8.5 \%$ is reported. Risk factors for SNE include transplant from allogeneic donors, severe acute graft vs host disease, total body irradiation, and treatment with cyclosporine A (CSA). CSA toxicity is the most common neurologic event, with an incidence of $11 \%$ among all allogeneic transplant recipients, and $17 \%$ if only unrelated hematopoietic stem cell transplant (HSCT) recipients are considered. CSA toxicity is usually reversible when CSA is discontinued. SNE caused by radio/chemotherapy, CNS infections, brain hemorrhage, or immune-mediated complications of HSCT are rare events.

\section{COGNITIVE EFFECTS AND MECHANISMS OF LEAD TOXICITY}

The effects of lead on the cognitive development of children, behavioral effects, reasons for the child's exquisite sensitivity, and the long-term prognosis of lead toxicity are reviewed at the Center for Trace Element Studies and Environmental Neurotoxicology, Staten Island, NY. The direct neurotoxic effects of lead include apoptosis, and damage to 